\title{
Relation between State-Selected or State-Averaged Cross Sections of Endothermic Reactions and Rate Constants of Exothermic Reactions. Application of Bimolecular Microcanonical Activated Complex Theory*
}

\author{
R. A. Marcus \\ Noyes Chemical Laboratory, University of Illinois, Urbana, Illinois 61801
}

(Received 16 February 1970)

\begin{abstract}
An expression is derived relating state-selected or state-averaged molecular beam reaction cross sections of endothermic reactions to observed rate constants for the inverse (exothermic) reactions. An approximation of Anlauf, Maylotte, Polanyi, and Bernstein is used, together with bimolecular microcanonical activated complex theory [R. A. Marcus, J. Chem. Phys. 45, 2138 (1966)].
\end{abstract}

\section{INTRODUCTION}

In a previous paper ${ }^{1}$ a microcanonical ensemble of reacting pairs of molecules in bimolecular reactions was considered and a quasiequilibrium assumed between the pairs and activated complexes moving in a positive direction along the reaction coordinate. ${ }^{2}$ As noted previously, this microcanonical version of activated complex theory is related to the usual version by means of a Laplace transform. ${ }^{3}$

Recently, in an interesting and most stimulating Communication, Anlauf, Maylotte, Polanyi, and Bernstein $^{4}$ have calculated rates of formation of a particular vibrational-rotational-translational state (some most probable state) of products of an endothermic reaction, as a function of initial quantum state of reactants at fixed total energy. They used infrared chemiluminescence data on the (inverse) exothermic reaction, plus microscopic reversibility and two approximations, including one to describe the most probable state.

In the present paper we employ one of their assumptions, together with microcanonical activated complex theory, to calculate molecular beam reaction total cross sections of an endothermic reaction, as a function of the initial vibrational-rotational-translational state in the incident beam, using rate constants of the (inverse) exothermic reaction [Eq. (11)]. An expression is also given for a velocity-selected internal state-averaged cross section [Eq. $\left(11^{\prime}\right)$ ] since in practice the beams are not usually state-selected. The relation of Eq. (11) to the results of Ref. 4 is described in a subsequent section.

Previously, bimolecular microcanonical activated complex theory ${ }^{1}$ was used to formulate a reaction crosssection theory $y^{5}$ (as a function of initial quantum state) for a different class of reactions, "nearly vibrationally adiabatic" ones. It was also used, ${ }^{1}$ in conjunction with available computer data, to test certain aspects of activated complex theory. In both cases the theory was in good agreement with the computer results without introducing adjustable parameters. ${ }^{1,6}$ The results in Ref. 1 have been illustrated by application to specific cases recently. ${ }^{7}$

\section{THEORY}

If a rotational-vibrational state of reactants $\mathrm{C}+\mathrm{D}$ in an endothermic reaction

$$
\mathrm{C}+\mathrm{D} \rightarrow \mathrm{A}+\mathrm{B} \quad \text { (endothermic) }
$$

is denoted by $n^{\prime}$ and that of the products $\mathrm{A}+\mathrm{B}$, by $n$, then the equation of microcanonical activated complex theory can be written in the present case as ${ }^{8}$

$$
\begin{aligned}
\sum_{n^{\prime}} k^{\prime 2} g^{\prime}\left(n^{\prime}\right) \sum_{n} \sigma^{\prime}\left(n k \mid n^{\prime} k^{\prime}\right) & =\sum_{n} k^{2} g(n) \sum_{n^{\prime}} \sigma\left(n^{\prime} k^{\prime} \mid n k\right) \\
& =\pi N^{\ddagger}(E),
\end{aligned}
$$

where the cross sections $\sigma^{\prime}$ and $\sigma$ are averages over the spatial degeneracy of the rotational angular-momentum states; $k=\mu V / \hbar, k^{\prime}=\mu^{\prime} V^{\prime} / \hbar$, and the primes refer throughout to the properties of $\mathrm{C}+\mathrm{D}\left[\mu^{\prime}=\right.$ reduced mass, $V^{\prime}$ is the initial relative translational velocity, $g^{\prime}\left(n^{\prime}\right)$ is the rotational state degeneracy, $n^{\prime}$ is the totality of internal state quantum numbers, apart from those describing the rotational degeneracy of $\mathrm{C}$ and that of $\mathrm{D}, \sigma^{\prime}$ is the reaction cross section for $\left.n^{\prime} k^{\prime} \rightarrow n k\right]$. Unprimed quantities refer to properties of $A+B$. Summations in (2) are over all states consistent with a total energy $E ; N^{\ddagger}(E)$ is the number of quantum states of the activated complex with total energy equal to or less than $E$.

By examination of classical trajectories for the exothermic reaction

$$
\mathrm{A}+\mathrm{B} \rightarrow \mathrm{C}+\mathrm{D} \quad \text { (exothermic), }
$$

the authors of Ref. 4 suggested that the distribution of states $n^{\prime}$ produced in Reaction (3) was approximately independent of the initial distribution of $\mathrm{A}+\mathrm{B}$ states, $k n$, at any $E$. It would then follow that the cross section $\sigma\left(n^{\prime} k^{\prime} \mid n k\right)$ for Reaction (3) is proportional to a factor (denoted here by $\kappa^{n^{\prime}}$ ) dependent on $n^{\prime} k^{\prime}$ and one dependent on $n k$. On normalizing by $\kappa^{n^{\prime}}$ by summing over all final states $n^{\prime}$ at the given $E$ in Reaction (3) it follows that

$$
\sigma\left(n^{\prime} k^{\prime} \mid n k\right) / \sum_{n^{\prime}} \sigma\left(n^{\prime} k^{\prime} \mid n k\right)=\kappa^{n^{\prime}}, \quad \sum_{n^{\prime}} \kappa^{n^{\prime}}=1,
$$

where the summation is over all states consistent with the given $E$.

The desired total cross section for the endothermic reaction (1) from a state $n^{\prime} k^{\prime}$ of $\mathrm{C}+\mathrm{D}$ is denoted by $\sigma^{\prime}\left(n^{\prime} k^{\prime}\right)$. It equals

$$
\sigma^{\prime}\left(n^{\prime} k^{\prime}\right)=\sum_{n} \sigma^{\prime}\left(n k \mid n^{\prime} k^{\prime}\right)
$$


The second equality in (2), together with (4), yields

$$
\sum_{n} k^{2} g(n) \sigma\left(n^{\prime} k^{\prime} \mid n k\right)=\pi N^{\ddagger}(E) \kappa^{n^{\prime}} .
$$

The last two equations and microscopic reversibility. ${ }^{9}$

$$
k^{2} g(n) \sigma\left(n^{\prime} k^{\prime} \mid n k\right)=k^{\prime 2} g^{\prime}\left(n^{\prime}\right) \sigma^{\prime}\left(n k \mid n^{\prime} k^{\prime}\right),
$$

yield an expression for $\sigma^{\prime}\left(n^{\prime} k^{\prime}\right)$ :

$$
\sigma^{\prime}\left(n^{\prime} k^{\prime}\right)=\pi N^{\ddagger}(E) \kappa^{n^{\prime}} /\left[k^{\prime 2} g^{\prime}\left(n^{\prime}\right)\right] \text {. }
$$

$\kappa^{n \prime}$ is readily expressed in terms of known quantities, as follows: The rate constant of the exothermic reaction (3), to form C and D in state $n^{\prime}$, is denoted by $k_{R}{ }^{\prime \prime}$. Throughout this paper, rate constants will be designated by $R$ subscripts. $k_{R}{ }^{\prime}$ equals $V \sigma\left(n^{\prime} k^{\prime} \mid n k\right)$, suitably averaged over $n$ and $k^{10}$ :

$$
\begin{aligned}
& k_{R^{n}}{ }^{\prime}=\sum_{n} g(n) \int_{E=0}^{\infty} V \sigma\left(n^{\prime} k^{\prime} \mid n k\right) \\
& \times \exp \left(-E / k_{B} T\right) k^{2} d k / 2 \pi^{2} Q
\end{aligned}
$$

where $k_{B}$ and $Q$ are Boltzmann's constant and the rotational-vibrational- (and in center-of-mass system) translational partition function of $A+B$, for unit volume. The over-all rate constant $k_{R}$ of the exothermic Reaction (3) is

$$
k_{R}=\sum_{n^{\prime}} k_{R}^{n^{\prime}}
$$

It follows from (4) and the last two definitions that

$$
\kappa^{n^{\prime}}=k_{R}{ }^{n^{\prime}} / k_{R}
$$

which, together with (8), yields the final result for the cross section of Reaction (1) :

$$
\sigma^{\prime}\left(n^{\prime} k^{\prime}\right)=\pi N^{\ddagger}(E)\left[k_{R}^{n^{\prime}} / k_{R}\right] /\left[k^{\prime 2} g^{\prime}\left(n^{\prime}\right)\right] .
$$

Equation (11) refers to state-selected incident beams. When, as is normally the case, the beams are velocityselected but internal-state averaged, the comparison of the measured and calculated cross sections would be made for a cross section averaged over $n^{\prime}, \sigma^{\prime}\left(k^{\prime}\right)$, as a function of $k^{\prime}\left(k^{\prime}=\mu^{\prime} V^{\prime} / \hbar\right)$ :

$$
\begin{aligned}
\sigma^{\prime}\left(k^{\prime}\right) & =\sum_{n^{\prime}} \sigma^{\prime}\left(n^{\prime} k^{\prime}\right) p^{\prime}\left(n^{\prime}\right) \\
& =\pi \sum_{n^{\prime}} N^{\ddagger}\left(E_{k^{\prime}}+E_{n^{\prime}}\right)\left[k_{R^{n^{\prime}}} / k_{R}\right]\left[p^{\prime}\left(n^{\prime}\right) / k^{\prime 2} g^{\prime}\left(n^{\prime}\right)\right],
\end{aligned}
$$

where $E_{k^{\prime}}$ is the translational energy in the center-ofmass system, $E_{n}$, is the vibrational-rotational energy of state $n^{\prime}$ of $\mathrm{C}+\mathrm{D}$, and $p^{\prime}\left(n^{\prime}\right)$ is the rotational-vibrational distribution function of $\mathrm{C}$ and $\mathrm{D}$ in the incident beams. When the internal-state distribution in the incident beams (or beam) is an equilibrium one, we have

$$
\frac{p^{\prime}\left(n^{\prime}\right)}{g^{\prime}\left(n^{\prime}\right)}=\frac{\exp \left(-E_{n^{\prime}} / k_{B} T\right)}{\left(Q_{\text {int }}{ }^{\prime}\right)},
$$

$Q_{\text {int }}{ }^{\prime}$ being the product of the rotational-vibrational partition functions of $C$ and $D$.
Expressions for $N^{\ddagger}(E)$ in (11), and hence for $N^{\ddagger}\left(E_{k^{\prime}}+E_{n^{\prime}}\right)$ in $\left(11^{\prime}\right)$, are given later, but first we compare Eq. (11) with the results in Ref. 4.

\section{RELATION TO THE INVESTIGATION OF ANLAUF, MAYLOTTE, POLANYI, AND BERNSTEIN}

Since the rate constants $k_{R}{ }^{\prime \prime}$ in Reaction (3) to yield state $n^{\prime}$ of $\mathrm{C}+\mathrm{D}$ were Boltzmann averages of $V \sigma\left(n^{\prime} k^{\prime} \mid n k\right) \quad$ [Eq. (9)], a "microcanonical rate constant" to form state $k n$ from state $k^{\prime} n^{\prime}$ in Reaction (1) can be defined in a quite natural manner as

$$
k_{R}{ }^{\prime}\left(n k \mid n^{\prime} k^{\prime}\right)=V^{\prime} \sigma^{\prime}\left(n k \mid n^{\prime} k^{\prime}\right) .
$$

This rate constant depends on $n^{\prime} k^{\prime} V^{\prime}$, and so depends on the total energy. Equation (12) together with microscopic reversibility [Eq. (7)], the approximation in Eq. (4), and Eq. (10) yield

$$
\begin{aligned}
& k_{R}{ }^{\prime}\left(n k \mid n^{\prime} k^{\prime}\right)=\left[g(n) / g^{\prime}\left(n^{\prime}\right)\right]\left(V^{\prime} k^{2} / V k^{\prime 2}\right) \\
& \times\left[k_{R}^{n^{\prime}} / k_{R}\right] V \sum_{n^{\prime}} \sigma\left(n^{\prime} k^{\prime} \mid n k\right) .
\end{aligned}
$$

When Eq. (13) is specialized to the case of an atom plus diatomic molecule, and when $n k$ is replaced by some "most probable" value, $\hat{n} k$, and $\sum_{n} \hat{V}_{\sigma}\left(n^{\prime} k^{\prime} \mid \hat{n} \hat{k}\right)$ then replaced by $k_{R}$, Eq. (2) of Anlauf, Maylotte, Polanyi, and Bernstein is obtained. ${ }^{11}$ For $\hat{n}$ a thermal equilibrium value was assumed, and for $k$ a value was used which, in effect, treated the relative translational motion as being the effective motion in overcoming the barrier. ${ }^{4}$ [For the main purpose of these authors, namely, obtaining relative values of $k_{R}^{\prime}\left(\hat{n} \hat{k} \mid n^{\prime} k^{\prime}\right)$ as a function of $n^{\prime}$ and $k^{\prime}$, no assignment for $\hat{n}$ and $k$ was needed.] A rigorous definition of the true $\hat{n} \hat{k}$ can be obtained by defining it to be the implicit solution of the equation

$$
\hat{V} \sum_{n^{\prime}} \sigma\left(n^{\prime} k^{\prime} \mid \hat{n} \hat{k}\right)=\left\langle V \sum_{n^{\prime}} \sigma\left(n^{\prime} k^{\prime} \mid n k\right)\right\rangle
$$

for then it would follow that ${ }^{12}$

$$
k_{R}{ }^{\prime}\left(\hat{n} \hat{k} \mid n^{\prime} k^{\prime}\right)=\left[g(\hat{n}) / g^{\prime}\left(n^{\prime}\right)\right] k_{R^{n}}{ }^{\prime}\left[\hat{k} \mu / k^{\prime} \mu^{\prime}\right],
$$

which is equivalent to Eq. (2) of Ref. 4. One sees from (14) that $\hat{n}$ and $k$ do not depend on $n^{\prime}$ but do depend on the total energy $E$. (The summations over $n^{\prime}$ are at constant $E$.) The solution of (14) for $\hat{n}$ and $\hat{k}$ would be formidable, except for simple cases, such as one which assumes $\sigma\left(n^{\prime} k^{\prime} \mid n k\right)$ to be independent of $n$.

The relation between (12) and (13) on one hand, and Eq. (11) on the other is readily established: When (12) and (13) are summed over all $n$ at the given $E$, and Eqs. (4) and (6) introduced, Eq. (11) is obtained.

Comparison of (11) and (15) shows that the former

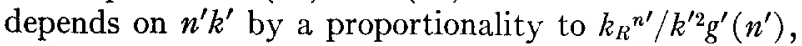
while the second has a proportionality to $k_{R^{n}}{ }^{\prime} / k^{\prime} g^{\prime}\left(n^{\prime}\right)$. Thus, the plots in Ref. 4 for the relative values of $k_{R}{ }^{\prime}\left(\hat{n} \hat{k} \mid n^{\prime} k^{\prime}\right)$, when multiplied by a factor proportional to $1 / k^{\prime}$, immediately provide plots of the relative values of the molecular beam reaction cross section $\sigma^{\prime}\left(n^{\prime} k^{\prime}\right)$. 
Equation (2) of Ref. 4, and hence Eq. (15) above, are restricted to calculations at constant total $E$, the calculations being made for different relative amounts of $E_{n}$, and $E_{k^{\prime}}$. Equations (11) and $\left(11^{\prime}\right)$ are not restricted in this manner, since $n^{\prime}$ and $k^{\prime}$ are independently variable there. To use (11) or $\left(11^{\prime}\right)$ to calculate absolute values of $\sigma^{\prime}\left(n^{\prime} k^{\prime}\right)$ or $\sigma^{\prime}\left(k^{\prime}\right)$ it is necessary to know $k_{R^{n}}{ }^{\prime} / k_{R}$ and to calculate $N^{\ddagger}(E)$.

\section{PARTICULAR EXAMPLES OF $N^{\ddagger}(E)$ IN EQ. (11)}

In Eq. (11), when $k_{R^{n}} / k_{R}$ is measured for Reaction (3), only $N^{\ddagger}(E)$ remains to be determined. Different models of the activated complex give different values of $N^{\ddagger}(E)$ and hence different predicted values for $\sigma^{\prime}\left(n^{\prime} k^{\prime}\right)$. As usual, the measurement of the pre-exponential factor and activation energy of $k_{R}$ for Reaction (3) provides some insight into the activated complex and, hence, into $N^{\ddagger}(E)$.

The number of vibrational and vibrational-rotational states of energy-rich molecules has been calculated by a variety of methods in the case of unimolecular reactions. ${ }^{13}$ Analogous methods can be employed to evaluate $N^{\ddagger}(E)$ in bimolecular reactions. For example, the partition function of the activated complex in the center-of-mass system is

$$
Q^{\ddagger}=\sum_{n \ddagger} \exp \left(-E_{n}^{\ddagger} / k_{B} T\right),
$$

where the summation is over all rotational-vibrational states of the activated complex (degenerate ones included). The number of such states with energy equal to or less than $E_{n}{ }^{\ddagger}$ is denoted by $n^{\ddagger}\left(E_{n \ddagger}\right)$; i.e.,

$$
n^{\ddagger}\left(E_{n \ddagger}\right)=N^{\ddagger}(E),
$$

where the relation between $E_{n \ddagger}$ and $E$ is given later by Eq. (22).

$n^{\ddagger}\left(E_{n \ddagger}\right)$ is a step function, but in the sense of a Stieltjes integral Eq. (16) can be written as

$$
Q^{\ddagger}=\int_{E_{n} \ddagger=0}^{\infty} \frac{d n^{\ddagger}\left(E_{n}^{\ddagger}\right)}{d E_{n}^{\ddagger}} \exp \left(-\frac{E_{n}^{\ddagger}}{k_{B} T}\right) d E_{n}^{\ddagger} .
$$

$d n^{\ddagger}\left(E_{n}^{\ddagger}\right) / d E_{n}^{\ddagger}$ contains delta functions at each step of $n^{\ddagger}\left(E_{n}^{\ddagger}\right)$. Equation (18) yields

$Q^{\ddagger}=\left(k_{B} T\right)^{-1} \int_{E_{n} \ddagger=0}^{\infty} n^{\ddagger}\left(E_{n}^{\ddagger}\right) \exp \left(-\frac{E_{n}^{\ddagger}}{k_{B} T}\right) d E_{n}^{\ddagger}$

on integration by parts [since $n^{\ddagger}\left(E_{n}^{\ddagger}\right)$ vanishes at $\left.E_{n}^{\ddagger}=0\right]$. If $\left(k_{B} T\right)^{-1}$ is denoted by $s$ and (19) treated as a Laplace transform, ${ }^{14,15}$ inversion yields

$$
n^{\ddagger}\left(E_{n}^{\ddagger}\right)=(2 \pi i)^{-1} \int_{c-i \infty}^{c+i \infty} \frac{Q^{\ddagger}}{s} \exp \left(s E_{n}^{\ddagger}\right) d s .
$$

The total energy $E$ in Eq. (17) can be written as

$$
\begin{aligned}
E & =E_{n}+k^{2} \hbar^{2} / 2 \mu \\
& =E_{n^{\prime}}+k^{\prime 2} \hbar^{2} / 2 \mu^{\prime}+\Delta V_{0} \\
& =E_{n \ddagger}+V_{p}^{\ddagger}+k^{\ddagger 2} \hbar^{2} / 2 \mu^{\ddagger},
\end{aligned}
$$

where $V_{T}^{\ddagger}$ is the potential energy of the activated complex $^{16}$ (relative to that of the most stable configuration of $A+B$ taken as zero), $\Delta V_{0}$ is the change of potential energy for Reaction (3), and the $E_{n \ddagger}$ are the energy levels of the activated complex; $\mu^{\ddagger}$ and $k^{\ddagger}$ are the effective mass and wavenumber for motion of the latter along the reaction coordinate. The maximum accessible value of $E_{n \ddagger}$ at a total energy $E$ is the largest value consistent with $E-V_{p}{ }^{\ddagger}$ :

$$
E_{n \ddagger}(\max ) \leq E-V_{p}{ }^{\ddagger} .
$$

In activated complex theory, $V_{F}^{\ddagger}$ is obtained from the activation energy of Reaction (3) at $0^{\circ} \mathrm{K}, E_{a}{ }^{0}$, or that at temperature $T, E_{a}{ }^{17}$ :

$$
\begin{aligned}
& E_{a}^{0}=V_{p}^{\ddagger}+E_{0}^{\ddagger}-E_{0}, \\
& E_{a}=V_{p}^{\ddagger}+E_{0}^{\ddagger}-E_{0}+k T+\left\langle E_{n \ddagger}-E_{0 \ddagger}\right\rangle-\left\langle E_{n}-E_{0}\right\rangle,
\end{aligned}
$$

where the $E_{0}$ 's are the relevant vibrational zero-point energies and the averages are thermal averages.

For concreteness two common simple limiting models and the resultant $N^{\ddagger}(E)$ 's will be considered in the present section. These particular models are the loose and rigid activated complexes, ${ }^{18}$ the former being associated with reactions having unit collision efficiency when the activation plus centrifugal barrier is exceeded by the radial portion of the translational energy of relative motion. The second activated complex is associated with reactions have appreciable steric effects (found when rotations of the reactants becoming bending modes of the activated complex).

When the activated complex is a loose one, the usual expression written for $Q^{\ddagger}$ [when divided by $s$ for use in (20)] is ${ }^{16}$

$$
Q^{\ddagger}(s) / s=\left(2 I^{\ddagger} / \sigma^{\ddagger} \hbar^{2} s^{2}\right) Q_{R}^{\ddagger}(s) Q_{V^{\ddagger}}^{\ddagger}(s),
$$

where $s$ is $\left(k_{B} T\right)^{-1} ; I^{\ddagger}$ is $\mu R^{\ddagger 2}, R^{\ddagger}$ being the separation distance of A and B in the activated complex; $\sigma^{\ddagger}$ is 2 if $\mathrm{A}$ and $\mathrm{B}$ are identical, and unity otherwise; $Q_{R}^{\ddagger}(s)$ is the product of the rotational partition functions of $\mathrm{A}$ and $\mathrm{B}$, and $Q_{V}^{\ddagger}(s)$ is the product of their vibrational partition functions. The corresponding equation for the rigid activated complex is given later in Eq. (31).

Most of the remaining discussion in this section is given for concreteness and completeness. More detailed discussions are found in Refs. 13,14, 19, and 20. According to Eq. (20) applied to $Q_{R}^{\ddagger}$ and $n_{R}^{\ddagger}$, the inverse transform of $Q_{R}{ }^{\ddagger}(s) / s$ is the number of rotational states $n_{R}^{\ddagger}\left(E_{R}^{\ddagger}\right)$ with rotational energy less than $E_{R}^{\ddagger}$. Similarly, $Q_{V}^{\ddagger}(s) / s$ is the number of vibration states $n_{V}^{\ddagger}\left(E_{V}^{\ddagger}\right)$ with vibrational energy less than $E_{V}^{\ddagger}$. The minimum value of $E_{V}^{\ddagger}$ is $E_{0}^{\ddagger}$, the zero-point energy of the activated complex. Application of the convolution 
theorem to (20) and (24) yields

$$
\begin{aligned}
N^{\ddagger}(E) & =n^{\ddagger}\left(E_{n}^{\ddagger}\right) \\
& =\left(2 I^{\ddagger} / \sigma^{\ddagger} \hbar^{2}\right) \int_{0}^{E_{n} \ddagger} n_{V}^{\ddagger}\left(E_{V}^{\ddagger}\right) n_{R}^{\ddagger}\left(E_{n}^{\ddagger}-E_{V}^{\ddagger}\right) d E_{V}^{\ddagger}
\end{aligned}
$$

where, in virtue of $(22)$ and of treating $E_{n}{ }^{\ddagger}$ now as a "continuous" variable, we have

$$
E_{n}^{\ddagger}=E-V_{p^{2}}^{\ddagger}
$$

For an atom plus diatomic molecule, $Q_{R}^{\ddagger} / s$ equals $\left(2 I / \sigma_{a} \hbar^{2} s^{2}\right)$, where $I$ and $\sigma_{a}$ are the moment of inertia and symmetry number for the diatomic molecule. Thus, inversion via (20), applied now to $n_{R}^{\ddagger}$ and $Q_{R}^{\ddagger} / s$, yields ${ }^{21}$

$$
\begin{aligned}
n_{R}^{\ddagger}\left(E_{n}^{\ddagger}-E_{V^{\ddagger}}\right) & =0 & & \left(E_{n}^{\ddagger}<E_{V}^{\ddagger}\right) \\
& =\left(2 I / \sigma_{a} \hbar^{2}\right)\left(E_{n}^{\ddagger}-E_{V^{\ddagger}}\right) & & \left(E_{n}^{\ddagger}>E_{V}^{\ddagger}\right) .
\end{aligned}
$$

If, instead, $\mathrm{A}$ and $\mathrm{B}$ are, say, nonlinear polyatomic molecules, $Q_{R}^{\ddagger} / s$ is $\left(A_{x} A_{y} A_{z} B_{x} B_{y} B_{z}\right)^{1 / 2}\left(8 \pi / \sigma_{a} \sigma_{b} \hbar^{6}\right) / s^{4}$, where the $A$ 's and $B$ 's are principal moments of inertia of $\mathrm{A}$ and of $\mathrm{B}$, and $n_{R}\left(E_{n}-E_{V}^{\ddagger}\right)$ equals this coefficient of $s^{-4}$, multiplied by $\left(E_{n}-E_{V^{\ddagger}}\right)^{4} / 4$ !.

When $E$ is small enough that no vibrational excitation of the activated complex occurs, only the leading term in $Q_{v}{ }^{\ddagger} / s$ contributes, namely $s^{-1} \exp \left(-s E_{0}^{\ddagger}\right)$ for a nondegenerate lowest vibrational state. Inversion via (20) yields the expected result,

$$
\begin{aligned}
n_{V}^{\ddagger}\left(E_{V}^{\ddagger}\right) & =0 & & \left(E_{V}^{\ddagger}<E_{0}^{\ddagger}\right) \\
& =1 & & \left(E_{V}^{\ddagger}>E_{0}^{\ddagger}\right),
\end{aligned}
$$

and (25) and (26) yield, for example, for an atom plus diatomic molecule,

$$
N^{\ddagger}(E)=\left(2 I^{\ddagger} / \hbar^{2}\right)\left(2 I / \sigma_{a} \hbar^{2}\right)\left(E-V_{p}^{\ddagger}-E_{0}^{\ddagger}\right)^{2} / 2
$$

$\left(\sigma^{\ddagger}=1\right.$ here $)$.

If, instead, the energy $E$ is high enough for vibrational excitation of the activated complex to occur, more general expressions than (28) must be used, for example, ${ }^{19}$

$$
n_{V}^{\ddagger}\left(E_{V^{\ddagger}}^{\ddagger}\right)=\left(E_{V}^{\ddagger}-E_{0}^{\ddagger}+a E_{0}^{\ddagger}\right)^{M} /\left(M ! \prod_{i=1}^{M} h v_{i}^{\ddagger}\right)
$$

where $M$ is the number of vibrational degrees of freedom (frequencies $\nu_{i}^{\ddagger}$ ) of the activated complex, and $a$ is unity at high $E_{V}^{\ddagger}$ and a known function of $E_{V}^{\ddagger}$ at lower $E_{V}^{\ddagger} \cdot^{19}$ Many comparisons of (30) with exact computations are available..$^{20}$

In case of a linear rigid activated complex, the rotational partition function is $2 I^{\ddagger} / \sigma^{\ddagger} \hbar^{2} s$, while for a nonlinear rigid activated complex it is $\left(8 \pi I_{x}^{\ddagger} I_{y}^{\ddagger} I_{z}^{\ddagger}\right)^{3 / 2} / \hbar^{3} s^{3 / 2}$. Writing it in either case as $A_{R} / s^{q+1}$, where $q=0$ in the first instance and $\frac{1}{2}$ in the second, and where $A_{R}$ is the relevant constant, one has

$$
\frac{Q^{\ddagger}(s)}{s}=\frac{\left(A_{R} / s^{q+1}\right) Q_{V}^{\ddagger}(s)}{s} .
$$

Inversion via (20) and the convolution theorem yields

$$
\begin{aligned}
N^{\ddagger}(E) & =n^{\ddagger}\left(E_{n}^{\ddagger}\right) \\
& =A_{R} \int_{0}^{E_{n \ddagger}}\left(E_{n}^{\ddagger}-E_{V}^{\ddagger}\right)^{q_{n}} n_{V}^{\ddagger}\left(E_{V}^{\ddagger}\right) d E_{V}^{\ddagger} / \Gamma(q+1)
\end{aligned}
$$

where $E_{n}{ }^{\ddagger}$ is given by (26), and $\Gamma$ is the gamma function. When there is no vibrational excitation, $n_{V}^{\ddagger}\left(E_{V}^{\ddagger}\right)$ is given by (28), and integration of (32) yields

$$
N^{\ddagger}(E)=\left(E-V_{p}^{\ddagger}-E_{0}^{\ddagger}\right)^{q+1} A_{R} / \Gamma(q+2) .
$$

At high $E_{n}^{\ddagger}$, Eq. (30) can be introduced for $n_{V}^{\ddagger}$ into (32).

\section{ACKNOWLEDGMENTS}

I am particularly indebted to J. C. Polanyi and R. B. Bernstein for their very helpful correspondence and suggestions, and for transmitting to me their results in advance of publication. It is also a pleasure to acknowledge helpful discussions with Dr. Wing Wong of this laboratory.

* Acknowledgment is made to the donors of the Petroleum Research Fund, administered by the American Chemical Society, for partial support of this research. This research was also supported by a grant from the National Science Foundation.

${ }^{1}$ R. A. Marcus, J. Chem. Phys. 45, 2139 (1966).

2 A microcanonical ensemble for treating unimolecular reactions, on the other hand, is well known in the literature, being present in RRK theory (without "activated complex theory"), in RRKM theory [R. A. Marcus and O. K. Rice, J. Phys. Colloid Chem. 55, 894 (1951) ; R. A. Marcus, J. Chem. Phys. 20, 359 (1952) ] and, in a classical version, in the theory of mass spectra [H. M. Rosenstock, M. B. Walienstein, A. L. Wahrhaftig, and H. Eyring, Proc. Natl. Acad. Sci. U.S. 38, 667 (1952)].

${ }^{3}$ Reference 5 in Ref. 1.

4 K. G. Anlauf, D. H. Maylotte, J. C. Polanyi, and R. B. Bernstein, J. Chem. Phys. 51, 5716 (1969).

${ }^{5}$ R. A. Marcus, J. Chem. Phys. 45, 2630 (1966)

${ }^{6}$ R. A. Marcus, J. Chem. Phys. 46, 959 (1967).

${ }^{7} \mathrm{~K}$. Morokuma, B. C. Eu, and M. Karplus, J. Chem. Phys. $51,5193(1969)$.

${ }^{8}$ We use Eq. (3) of Ref. 1, with tunneling neglected and with any alternative reaction paths having similar complexes ( $\Gamma$ there is now a numerical factor rather than an operator) and with the degeneracy $g(n)$ being exhibited explicitly rather than contained in the summation. Another ensemble also discussed in Ref. 1 has both a given energy and a given total angular momentum [Eq. (5) there].

${ }^{9}$ For example, reviewed in J. Ross, J. C. Light, and K. E. Shuler, in Kinetic Processes in Gases and Plasmas, edited by A. R. Hochstim (Academic, New York, 1969), Chap. 8.

${ }_{10}$ For example, readily derived from expressions in Ref. 9 or in M. A. Eliason and J. O. Hirschfelder, J. Chem. 30, 1426 (1959).

"In making the comparison, it will be noted that our $k_{R}{ }^{\prime}\left(n k \mid n^{\prime} k^{\prime}\right)$ and $k_{R^{n \prime}}$ are the $k_{r}\left(v J \mid v^{\prime} J^{\prime} ; E_{\tau}^{\prime}\right)$ and $k_{f}\left(v^{\prime} J^{\prime}\right)$ of Ref. 4. Also, there, their $\sigma_{R}\left(v^{\prime} J^{\prime} \mid v J ; E_{T}\right)$ and $\sigma_{R}\left(v J \mid v^{\prime} J^{\prime} ; E_{\tau^{\prime}}\right)$ (abbreviated by $\sigma_{f}$ and $\left.\sigma_{r}\right)$ are our $\sigma\left(n^{\prime} k^{\prime} \mid n k\right)$ and $\sigma^{\prime}\left(n k \mid n^{\prime} k^{\prime}\right)$, respectively.

${ }^{12}$ For example, the average, \langle\rangle , on the right side of (14) is over all $n$ and $k$, thus making it $k$. Equation (13), with $n k=\hat{n} \hat{k}$, then yields (15). 
${ }^{13}$ Reviewed in W. Forst and Z. Prasil, J. Chem. Phys. 51, 3006 (1969).

${ }^{14}$ E. Thiele, J. Chem. Phys. 39, 3258 (1963); P. C. Haarhoff, Mol. Phys. 6, 337 (1963); 7, 101 (1963); C. Lifshitz and M. Wolfsberg, J. Chem. Phys. 41, 1879 (1964); W. Forst, Z. Prasil, and P. St. Laurent, ibid. 46, 3737 (1967) ; 47, 1431 (1968).

${ }^{15}$ S. H. Bauer, J. Chem. Phys. 6, 403 (1938); 7, 1097 (1939); C. Kittel, Elementary Statistical Physics (Wiley, New York, $1958)$, p. 57.

${ }^{16}$ The arguments are readily extended to the case where the position of the activated complex (and hence $V_{p} \ddagger$ ) depends on $n$, e.g., depends on the angular momentum via a centrifugal effect [cf., R. A. Marcus, J. Chem. Phys. 43, 2658 (1965) ; 52, 1018 (1907); D. L. Bunker and M. Pattengill, ibid. 48, 772 (1968); W. Forst, ibid. 48, 3665 (1968); E. V. Waage and B. S. Rabinovitch, Chem. Rev. (to be published)].

${ }^{17} \mathrm{By}$ definition, $E_{a}=-k T \partial \ln k_{R}^{\prime} / \partial(1 / T)$, and Eq. follows upon introduction of an activated complex theory expression for $k_{R}^{\prime}$.

18 Compare R. A. Marcus and O. K. Rice, Ref. 2.

${ }^{19} \mathrm{~B}$. S. Rabinovitch and R. W. Diesen, J. Chem. Phys. 30, $735(1959)$; B. S. Rabinovitch and J. H. Current, ibid. 35, 2250 (1961); G. Z. Whitten and B. S. Rabinovitch, ibid. 38, 2466 $(1963) ; 41,1883(1964)$. Our $E_{V}^{\ddagger}$ includes $E_{0}^{\ddagger}$, so that $E_{V}^{\ddagger}-E_{0}^{\ddagger}$ corresponds to the "nonfixed" vibrational energy (energy "not fixed as zero-point energy") used in Ref. 18 and by subsequent authors.

${ }^{20}$ For example, D. C. Tardy, B. S. Rabinovitch, and G. Z. Whitten, J. Chem. Phys. 48, 1427 (1968); Refs. 13 and 19.

${ }^{21}$ For $E_{R}^{\ddagger}$ in the interval between the lowest rotational state $(j=0)$ and the next one $j=1, n_{R}^{\ddagger}\left(E_{R}^{\ddagger}\right)$ should be unity. The discrepancy with (27) arises because of the use of a classical expression for $Q_{R}^{\ddagger}(s)$, but the error is normally negligible, because of the close spacing of the rotational energy levels in $Q_{R}{ }^{\ddagger}(s)$.

\title{
Quenching of Phosphorescence by Paramagnetic Molecules in Rigid Media. II. Quenching of Perdeuderated Naphthalene by $\mathrm{Co}^{++}, \mathrm{Cr}^{3+}, \mathrm{Cu}^{++}$, and $\mathrm{Ni}^{++*}$
}

\author{
C. O. Hitu $\dagger$ AND S. H. Lin \\ Department of Chemistry, Arizona State University, Tempe, Arizona 85281
}

(Received 22 December 1969)

\begin{abstract}
Experimental observation of the steady-state phosphorescence emission intensity of perdeuterated naphthalene as a function of paramagnetic quencher concentrations has been carried out in solid matrices of ethyl alcohol at liquid-nitrogen temperature. The paramagnetic quenchers chosen for study are the transition-metal ions, $\mathrm{Co}^{++}, \mathrm{Cr}^{3+}, \mathrm{Cu}^{++}$, and $\mathrm{Ni}^{++}$. The decay curves of phosphorescence are nonexponential, and the deviation from the exponentiality depends on the concentrations of quenchers. The experimental results are correlated and interpreted by using theoretical expressions derived from the Hoijtink's exchange interaction mechanism.
\end{abstract}

\section{INTRODUCTION}

A great number of experimental examples which illustrate the enhancement of a weak electronic transition in a molecule through the interactions with neighboring perturbers are now available. The spin-forbidden transitions of atomatic molecules in their excited triplet electronic states can be enhanced by their interaction with paramagnetic molecules or ions. ${ }^{1-10}$ Many of the investigations dealing with this type of quenching have used systems in which bimolecular quenching is controlled by the diffusion of interacting species. It has been shown ${ }^{11-13}$ that results of diffusion-controlled experiments cannot be used unambiguously to determine the relative quenching efficiencies for different molecules because of the difficulty arising from differences in their diffusion coefficients, lifetimes of the bimolecular complexes formed, and the relative quenching efficiencies of the complexes. To study the nature of the interaction mechanism of paramagnetic quenching, which is reflected in the intermolecular distance dependence of the interaction, one may measure the relative efficiencies with which the paramagnetic molecules or ions enhance the transitions (radiative and nonradiative) from the excited triplet state to the ground state from static experiments.
In our previous paper, ${ }^{14}$ we studied the quenching of phosphorescence by paramagnetic molecules or ions in rigid media from the viewpoint of Hoijtink's exchange mechanism, ${ }^{15-17}$ and for the purpose of correlating experimental data theoretical expressions for decay function, relative yield, mean duration, etc., ${ }^{14}$ of phosphorescence were derived as function of the concentration of paramagnetic quenchers. In this preliminary communication, we report the measurements of the effects of paramagnetic transition-metal ions $\left(\mathrm{Co}^{++}\right.$, $\mathrm{Cr}^{3+}, \mathrm{Cu}^{++}$, and $\mathrm{Ni}^{++}$) on the rate of perdeuterated naphthalene in solid matrices of $95 \%$ ethyl alcohol. Essentially, the experiments consist of the observation of the steady-state phosphorescent emission intensity as functions of added quencher. The results show that the decay curves are not exponential and the decay rate depends on the concentrations of paramagnetic ions. It will be shown that the derived expressions can correlate the experimental data very well.

\section{EXPERIMENTAL}

The perdeuterated naphthalene is obtained from International Chemical and Nuclear Corporation, and the quencher molecules chosen for study are the transition-metal ions, $\mathrm{Co}^{++}, \mathrm{Cr}^{3+}, \mathrm{Cu}^{++}$, and $\mathrm{Ni}^{++}$, from 